\title{
9
}

\section{Participant Observation}

Eric Laurier

Synopsis

Participant observation is a minimal method that, as its name suggests, involves participating and observing places, practices and people. It proceeds along two trajectories. The first trajectory is searching for a setting where the social or cultural thing that the researcher wishes to study is happening and becoming intimate with the group that populates that setting. The second trajectory, which moves in parallel with the first, is that the researcher themselves changes how they understand the setting and its inhabitants. By undertaking participant observation the researcher aims to gain the authority of 'insider' knowledge. They will then able to report on what matters for that culture, how things are organised and why locals do things in the distinctive ways that they do. Geographers have used it to study communities, public spaces, institutions, embodied practices, game playing, illness, political action, online communication, everyday life and other spatial phenomena.

The chapter is organized into the following sections:

- What is participant observation

- Legitimate peripheral participation

- From the outsider to the insider

- How to find a familiar thing interesting 
- Changing your grip

- Strengths and weaknesses

\section{WHAT IS PARTICIPANT OBSERVATION}

Participant observation is perhaps the easiest method in the world to use because it is ubiquitous and we can all already do it. From the moment we are born we are, in various ways, observing the world around us and trying to participate in it. Children, acquiring language for the first time, listen and watch what, when and how their parents are doing what they are doing. They observe greetings and have greetings directed at them, and attempt to participate by, at first, looking and, later, waving and making sounds that approximate, and eventually are, hellos and goodbyes. It is, of course, not just children who use this method to acquire skills: air traffic controllers spend a great deal of time observing air traffic control and are gradually entered into the practical demands of directing planes as fully participating air traffic controllers. International migrants, finding themselves in foreign countries, have the massive task of observing a multitude of activities and exactly how they are done in order to participate in new cultures. Amongst other background knowledge they have to acquire the locals' taken-for-granted ways of getting everyday things done, such as greetings, ordering coffee, queuing for buses, making small talk, paying their taxes and so on. 
Participant-observation is a method that is built out of two familiar parts: observation and participation. Observation of spatial phenomena has been a central method for geography from its very outset, whether it be observing the movement of glaciers or the traffic flows of cities. With a reasonable vantage point, the right tools, and tables for documentation researchers can begin to collect, compare and count the presence, movement and features of individuals, groups and populations in defined spaces. Participation is a form of involvement, or association, in a group, practice or event. For many students trained in the observational methods of the physical sciences the validity of their observations is based upon on distance and neutrality rather than participation. Combining observation with participation appears to risk destroying the quality of the data collected by this method. However the power of participant-observation lies in its intimacy with, and grounded perspective upon, the places, practices and people it studies. The knowledge acquired from this method is as much reliant on participation as it is observation. To drive this point home, frequently human geographers reverse the terms of the title of the method and call it observant participation.

Participant observation, as you are probably beginning to realise, requires us to reconsider the questions we can ask as geographers and to change our criteria for judging whether the method was adeptly executed or not. Typical indicators of 'scientific rigor' must be reconsidered for this approach, though this does not mean we abandon rigor. While we cannot test a hypothesis, we can change our perspective and question received opinions. While we cannot collect standardised data and keep our variables equal, we can reveal specifics, show similarities and uncover differences. In undertaking participant observation we can begin to collect what things are 
relevant to study for the situation we are participating in. We can explain to other researchers why those things are significant to the group or practice that we have been participating in. Most important of all, we can provide descriptions of how those ordinary and extraordinary things are accomplished by the people we are studying, with the equipment they have for living, situated in the varied environments that they inhabit. In sum, participant observation's has strengths in describing the local processes, practices, norms, values, reasoning, technologies and so on that constitute social and cultural lifeworlds.

To return briefly to the example of traffic flows, participant observation could be undertaken of any one of the groups that are involved in the production of traffic and which have different local understandings of traffic. You could spend time in the unfamiliar world of a traffic control room with engineers and planners to understand and be able to speak about how they manage traffic, and its disruptions (Gordon 2012). You could spend time in a more familiar world: accompanying commuters that drive through that traffic and begin to understand what their practices are, how they drive and why they take the routes that they do (Laurier et al 2008).

Participant observation is a long established method for engaging with familiar and unfamiliar life-worlds in geography but also anthropology and sociology. It is undertaken as a trajectory from one perspective to another which is most conspicuous in unfamiliar environments, such as a place, community, workplace or institution that you have not been part of before. Think of the effort and time required to do informative participant observation studies of UK motorway-traffic control (Gordon 2012), social workers urban outreach (Hall \& Smith 2013) or the French court system (Latour 2010). Yet while it is a struggle to observe what is happening in these places, let alone participate in them, your findings will be of value by the very 
fact that how many unfamiliar places are organized is not part of the everyday knowledge of members of society.

When participant observation is used in familiar settings such as shopping in the supermarket (Cochoy 2008), walking along a coastal path (Wylie 2005), or the rave scene in Goa (Saldanha, 1999) or riding an elevator (Hirschauer, 2005) then the researcher has to work all the harder to notice the things that happen in these settings that they usually overlook. In other words, in familiar situations the problem is how to change the way we observe them from what phenomenologists called the 'natural attitude'. As participants in these common cultures, they have become so familiar to us that as ordinary members of society we no longer spend time noticing what we find relevant and irrelevant in them, what our assumptions are about them and how we inhabit them.

Gender is a classic example of a seen but un-noticed feature of each society's common cultures that is open to study by participant observation. For men, how it is that they go about orienting toward and producing themselves as men in the local cultures where they are located, has been learnt from their earliest years onwards and, while reflected upon, is profoundly taken-for-granted. A participant observation study in the UK or USA, for instance, could then select one of the many sites where gender has added significance such as a gym and then spend time observing and participating in what it is that the male members do at the gym and how they go about it. Such a study also raises an abiding methodological problem for participant observation. Any individual researcher according to their existing gendering by society has both different claims to insider knowledge and limits to their participation. Age, race and a number of social divisions, that have been the focus of geographical research, pose similar problems for participant observation studies 
because the researcher has not only already given insider or outsider status for these social categories and cannot attain full membership of the social group that they are interested in. Even for more permeable social groups to what extent the participant is able to approach or be able to claim that they have an insider's perspective requires itself, careful analysis.

\section{LEGITIMATE PERIPHERAL PARTICIPATION}

We have then established that a key assumption of participant observation is that observing social activity is predicated participating, even in the most minimal of ways. However my students often want to label the short studies that they undertake 'non-participant observation' because they worry that they were not participating in the activities that they were observing. A common case is when students have studied a city street and it seems to them that they simply sat on a bench taking notes and were not involved in settings and its events. Are they then not non-participantobservers? No, because those very spaces accommodate our presence and give us a role; we change the spaces we are present in to greater or lesser degrees, even when we are seemingly passive. By our very presence we are participating members of the public, in a public space that provides persons using it with the right to do things like people-watching and to be watched by other people in return.

There are many other sites apart from public spaces which also allow us to participate in them in a minimal fashion while observing what happens. The education researchers Lave \& Wenger (1991) have provided a useful term to make sense of the position that you can occupy in these settings. They call the involvement of researchers in these settings 'legitimate peripheral participation'. While many places allow people to sit at their edges without having to become full participants, they do 
so in different ways and proffer different rights (which are also useful features of the place to describe and analyse). Offices allow new employees to shadow their staff, classrooms allow trainee teachers to sit-in on lessons and internet forums allow lurkers (though this latter term hints at the morality of observing without participating more fully). From these peripheral perspectives there are then informative findings that can be made about the cultures under study even if the researchers do not become insiders or competent members of the local cultures.

\section{FROM THE OUTSIDER TO THE INSIDER}

Having sketched out the validity of legitimate peripheral participation to undertake limited participant observations of certain groups or activities let us now turn to the aim that we began with: the outsider in their participation is becoming an insider and will change their perspective on the local world of the group that they are studying. That sounds rather grand, what it means more prosaically is that if you spend a couple of months working on a waste collection vehicle you will no longer look at the rubbish in the street in the same way, nor neighbourhoods themselves. You will have begun to acquire the rubbish collector's ways of perceiving, assessing and acting upon the state of people's rubbish bins. As we noted above, there are very likely be limits to becoming a legitimate member of the groups you are studying. For becoming a rubbish collector, the limits of becoming an insider may be subtle and lie around whether the researcher is covert or overt, where they find themselves positioned by members of the waste collection vehicle, whether they undertake ritual inductions, whether they have the abilities to undertake the job and more.

What you should be aiming to achieve when undertaking participation is to follow a trajectory from an 'outsider' to an 'insider', even if you do run up against the limits of doing so. Those limits are themselves, qualities of the place, practice or group that 
are open to analysis and should become part of the findings of your research. Despite there being no template for doing participant observation there are features in the course of its trajectory which, while not specifying what is to be done, will give you a sense of whether you are making any progress or not. You should be able to detect your own perspective changing as you begin to grasp how it is that people achieve the practices that they are doing and why they do so, may then also gradually become apparent. This is what Jack Katz $(2001 ; 2002)$ calls 'from how to why'. More importantly, the group around you will change how they categorise you as you shift from a 'newby' or a 'beginner' to whatever they call a competent and/or accepted member or practitioner. It may that you can become 'one of us' or that you are always only a partial member of the group as we noted earlier. Just how long it takes to become competent in or 'doing being' what you choose to study, and indeed whether it is possible to reach that state, will vary according to what it is. Are you trying to how to serve coffee in a local café or become a marathon swimmer or a leader of a drug gang?

Should you choose to study a supermarket as a shopper or stacking the shelves as a member of the staff then it is not so demanding to become competent in these activities. However, other communities of practice, such as urban gangs, dairy farmers or taxi drivers, may take considerable time and effort before you will be recognized as an accepted member of their groups. For whatever activity you decide to participate in there will be different ordinary and expert ways in which you are instructed in how to do it, from the more formal (i.e. lessons, workshops, courses, rulebooks, etc.) to the informal (tips, jokes, brief chats). However long the journey, the participant observer makes notes at all points to both provide a record and push themselves to reflect on what they are experiencing, how people are doing the things around them that they do, and, significantly, the shifts in their own perspective on 
the phenomena that they are studying. It is at heart a process of becoming attentive and acquiring a new perspective on some small part of the world.

\section{HOW TO FIND A FAMILIAR THING INTERESTING}

In studying the everyday it is hard not to exercise a kind of professional scepticism that subverts the intelligibility of the everyday thing that we are able to see as participants in a shared culture. The danger is that in trying to find a new perspective we go too far and act like a Martian who has landed on Earth. Such a creature is without the shared methods that we have for making sense of the things in our local environment. A central insight from phenomenology is that the intelligibility of our environment is tied to the fact that we are participants in a shared lifeworld and that, as noted earlier, we perceive with the linguistic categories we have been given by the acquisition of language.

In doing participant observation in places where we are already competent inhabitants and usually take the appearance of things for granted, the solution to doing adequate descriptions of them is, then, not to import strange Martian labels for the things we see or hear or otherwise sense almost instantly. Instead we aim to notice, uncover and analyse the categorisations of things that the locals do, and would, use to describe their observations.

If you are a 'local' and thus an 'insider' already you have a substantial advantage in providing adequate descriptions of how and why things get done in the way that they get done. Yet you are also at the equal disadvantage of no longer noticing how such things get done because they are so familiar as to be taken-for-granted, seen but 
unnoticed, and you may never have attempted to be interested in them. Consider the ordinary social and cultural categorisations of things that locals use. In a student's notes on the gym we find, for instance, that a 'a tired looking older woman' exchanged 'friendly' glances with 'another older woman', or that a 'teenage girl avoided the glance of another teenage boy'. The student is becoming attentive to how people use glances to do 'being friendly' or by not glancing back to do 'avoiding someone's attention'. The student begins to reflect on how as a competent member of a shared place, they are able to see what someone else sees. That is, they were able work out what what it was that the other person are looking at, not so much from pinpointing the exact direction of their look but by seeing what it is in the scene that they would glance at or who they would exchange glances with (e.g., the guy waiting the exercise machine is glancing at his watch, two members of the same social category 'older women' are exchanging glances).

The challenges of a participant observation will be different if you have pursued participant observations of new and/or uncommon sets of skills. Geographers have researched diverse sub-communities and events, such as historical re-enactments (M. Crang 1996), working in telebanking (Harper et al. 2000), Mexican women's labour conditions (Wright 2001), marathon swimming (Throsby 2013) and living among the mentally ill on the streets (Parr, 1998). For these more practically ambitious projects your adequacy as an observant participant turns on you having learnt things that other researchers, cannot be expected to know. This certainly makes delivering 'news' easier since, unlike 'exchanging glances' or 'answering the telephone' or 'buying a newspaper', not everyone knows how these more obscure, expert, secret, marginal or exotic cultures are organized and what their practices are and mean to them. Competence in performing the practices that constitute the place that you have selected to investigate will be one way in which your findings from participant 
observation will attain a reasonable degree of adequacy. Your aim is then, as it was for the familiar, to make available for further description, analysis and, under some circumstances, judgment, the practices and events that member of those places treat as unremarkable or routine.

\section{CHANGING YOUR GRIP}

Recording what you notice at all stages of doing participant observation is vital. If you are an outsider, then you will notice many peculiar practices at the beginning, practices that you may forget were noticeable by the time you are an insider. At the beginning you have the perspective of 'any person' - who may well be the audience you wish to write your report for at the end of your fieldwork - and you may find yourself offered a perspective by the setting itself as an 'incomer', a 'tourist', a 'guest' etc. As I have hinted at earlier, if you are a young adult studying children's cultures or the life an old folk's home then while your perspective and understanding will shift you will never become a child again nor will you yet be in your old age. Without keeping a record of your own struggles to understand what people do, how they do it and why they do it, you are likely to forget the perspective that you began with once you no longer have it. Consequently you will no longer appreciate what it is about local cultures that may seem odd, unfathomable or otherwise inaccessible to other researchers.

If you are already an insider, which is the case when studying many features of everyday life (though it may be that you have knowledge of an expert, exotic or esoteric practice) then the very process of recording should help you begin to see your taken-for-granted world afresh. Records, in the form mainly of notes, but also photographs and videos, will form your materials for thinking with when it comes to 
writing-up your participant observation. To write a powerful report on your participant observation requires documenting it as you go along because, not only will details slip away very quickly, your documenting process is the beginnings of your analysis.

Recording what you notice is not enough, of course, it needs to be coupled with techniques for helping the participant-observer open up their insider perspective for further inspection and reflection. The three techniques of note-taking for helping you get started that I will briefly describe here are: 1 . bracketing phenomena (see Box 1); 2. describing practices through instructions (see Box 2); 3. breaching norms and rules.

\section{Bracketing phenomena}

This technique for note-taking works most effectively when trying to record details of the familiar. Your ambition is to suspend your taken-for-granted knowledge of the thing you are trying to observe. In the example in Box 1 it was the common recognition of a cafe as empty. The recognisable state of affairs [empty] is bracketed, quite literally. It could be something of a less familiar kind such as how a manager at an organisation you have been studying has [the last word]. It could be, for skateboarders, doing an [ollie]. Once you have bracketed an ordinary phenomenon, your work as a participant-observer is to then to begin to describe how this state of affairs is produced, how it is recognised and begin to trace out then some of the implications it has for the group that use or populate the site.

\section{Box 1 The cafe is [empty]}

Staff can be present in the café but without customers the café is [empty]. What more is there to this easily recognizable state of affairs? There is: how the customer recognizes [empty] - which is bound up with the typical interior architectural construction of 
this café, and many others like it, which allows those entering to look around as they enter and see, at a glance, how busy the café is. It is bound up also with the expectations of time of day: 'this early hour' (e.g. around 7am) in this café known for its 'appearances as usual' on a weekday at this sort of time or as an environment of expectations. At the same time of day, an airport or flower market cafe is likely to be full. What the customer makes of [empty] is related to their orientation to the 'awakening' of the day. That is, the reasons for empty-ness are temporally located - it is 'just opened'. A customer is not put off or curious about this observable empty-ness during the opening time, the way they would be were it to be observably empty at $1 p m$ ('Why is it empty? Is the food bad? Are the staff rude? Is it expensive?'). (For more details see Laurier, 2008.)

Be wary when bracketing familiar features of the world that you do not then adopt the perspective of our Martian Invader. As an 'insider' you already have a store of practical knowledge about how the things you are observing function and what you are trying to do is open up, inspect and analyse your insider knowledge.

\section{Turning practices into instructions}

Where many other methods in geography struggle to provide information on the processual nature of systems, participant observation excels at describing how people do things, step-by-step. A technique to try in note-taking is, then, to transform what you are trying to describe into instructions or sequences of actions that would allow someone else to redo the thing somewhere else. If you are moving from outsider to insider this will be all the easier because the people around will be showing you how to do it or may actually pass you a list of instructions.

\section{Box 2 Taking a selfie}

Taking 'a selfie' photograph: I pick up my smartphone and take a few selfies. I break what I have just done into instructions: 1. Look at some other people's selfies. 2. Examine your current appearance. 3. Sort out hair, straighten specs etc. 4. 
Activate your camera app. 4. Hold your phone in your out-stretched arm (This is peculiarly difficult and worth returning to). 5 Examine the background as to whether it captures the place you want to be seen in (or is a distraction because your facial expression is the main focus here) 6. Produce a face (but what face? why?). 7. Try and match the timing of pushing the fire button with the face you wish to take (this is also peculiarly hard without losing the face because you are concentrating. I ended up clicking the button three or four times). 8. Examine the images produced on your camera. 9. Delete most of them. 10. Potentially redo the selfie on the basis of the earlier selfie. 11. Title the selfie (this leaves me thinking - what is this selfie doing, is it obvious, what else might be made of it? My initial list of instructions has not provided me with much by way of note yet and what I want to do next is then extend the descriptions of each step, to consider how each step relates to the next and observe how others are doing these steps. Briefly here then, looking at other people's selfies I see a range of possible facial expressions people can make and consider which of these are ones that 'do' something, is that what we want to 'do', for example, looking cool or looking daft or looking amazed. How does this relate to where my 'selfie' is, this was already something that came up when I was writing down the steps to produce a selfie. Am I at a major sports event, am I out with my friends, did my boyfriend just leave me. My appearance then should match what I am going to try and do with this selfie so people do not see me doing an 'amazed' expression because my boyfriend just left me. Or maybe they should. But then what is 'an amazed look' accomplishing and, as our analysis progresses what does this tell us about the social worlds in which selfies are produced.

In producing instructions we are trying to avoid producing a definition of the practice, or trying to explain why it is important, or what it means. We are accepting that there is a practice in the world that other members know how to recognise and may well know how to do. In disassembling it into instructions we begin to open up the practical knowledge that is required to do it. Once we can put ourselves into the middle of the action we can begin to make visible what shared problems emerge in this practice. For the example of the 'selfie' in Box 2, this meant briefly exploring the shared problem of exploring what self-taken public faces look like in different 
situations, and then, working with the camera to try and capture a face that fits and that will mean what we intended it to mean for others.

These basic instructions that you write down on first brush can then themselves be considered for further features of the phenomenon that they have missed. What can I add to this instruction for it to make more sense? Was it important who did the action? What were the expectations of the others? What are the criteria for success or failure? And so on. The additional questions and expansions that we make in response to rendering practices as instructions are ones that we will find from looking at other studies of the same or similar phenomena.

\section{Participation by breaching followed by observation}

Breaching experiments arise out of classic studies in the 1960s where social scientists, borrowing from other radical elements of 60 s culture, tried to disrupt social norms. They continue to serve a useful purpose for studying everyday settings because members of those settings use those very norms to make sense of the disruption.

\section{Box 3 Queueing a sandwich}

An exercise set for students by Nick Llewellyn at the University of Warwick was to disrupt the norms in supermarket checkouts by placing a sandwich on the floor at the end of a queue (line) in a busy supermarket. Students, having placed the sandwich (or similar ordinary item), would retire to a distance and keep notes on how the sandwich was dealt with. Customers arriving at the end of the queue would stand behind the sandwich and move it along in front of them, while the queue itself moved along. They would look at the customers ahead who might shrug or look around for the customers who had left the sandwich behind. The sandwich almost always made it to the point of sale where the checkout operator and neighbouring customers would discuss its status, often looking slightly concerned or 
frustrated. There were many points of analysis that could be made about what happened. Here we can note briefly how the details of the place that an item from the supermarket is found in is used to make sense of it. The sandwich placed by the students was treated, not as a sandwich for buying or that had simply fallen on to the floor, but by examining its proximity to the queue, it was seen as a placeholder for a shopper that for whatever reason had to depart the queue. Customers worked collectively to keep the absent shopper's place for them in the queue. In fact its removal was seen as a problem because it meant taking that person out of the queue.

The restrictions set in place by university ethics committees and procedures make some forms of breaching experiments harder to undertake. However mild forms of disruption, such as moving goods out of their usual locations in shops, (Box 3) which cause no harm and merely concern and consternation remain very useful for revealing taken-for-granted norms and rules. The design of breaching experiments has to be done with care so that it allows a variety of responses to the breach, has a plan for follow-on responses from the participant-observer and depending on the nature of the breaching, a de-briefing elements for the members of the setting where it is administered.

In analyzing what happens when the everyday practices in a particular place are disrupted we can look at, firstly, what the norms and rules are and, secondly, how they are used by members of different places. Planning the disruption will be the beginnings of this analysis because it forces us to change our perspective on how the place works and identify what its centrals features are that can then be breached. What breaching also brings to the surface is the particular ways that members of settings maintain and repair the normal appearances of the specific practices that is being disrupted. 


\section{STRENGTHS AND WEAKNESSES}

Human geography, like most other social sciences, has a host of classic topics such as power, class, race, identity and landscape. As big-picture issues they often sit uncomfortably with the more modest and small-picture concerns of actually doing participant observation. It is unlikely that you will solve problems that have dogged society and the social sciences for a century or more from your study of a drop-in centre, supermarket or airport arrivals gate. And yet, the use of participant observation should make you able to respecify what appear to be overpowering abstract problems into the shared practical problems of particular groups. It may be that in beginning to describe what happens at the local swimming pool your analysis begins uncover how masculinity and femininity are manifest in the pleasures (and sufferings) of sports practices (Throsby 2013). Or, in a participant observation of a workplace you may be able to show how the staff of multinational fast food companies resist, subvert or conform to the scripts their company expects them to follow (Leidner 1993).

Your results should be of the kind that are not pre-specificed by existing studies or theoretical accounts. Nor should you have been able to imagine them before doing your study. If either of these are the case then why bother using participant observation if you can say how the world works without ever consulting anything in the world.

The strengths of participant observation are:

- Establishing what is important for particular groups or for specific practices 
- Detailing how spatial activities and events are organised in terms that are recognisable to their members

- Understanding why specific groups or practices are carried out in the ways that they are

- Uncovering taken-for-granted aspects of the everyday and holding them up for scrutiny and analysis

- Requiring no technical knowledge, yet is challenging because it requires shifting to a new perspective

The weaknesses of participant observation are:

- It is not designed to for generalising beyond the event, group or practice you are studying. This does not mean that it cannot be profitably compared with other studies nor become the basis a study using other methods to check its generalisability but it does mean that participant observation's task is about findings that are specific and grounded.

- It cannot be used for hypothesis testing, though in another sense it should be about changing or challenging the assumptions that you began with

- It does not permit standardization of the phenomenon for comparison across multiple settings.

- Data is tied to the original researcher and can be challenging to deposit and share 
Participant observation allows you to build detailed descriptions from the ground up that should be recognizable to the groups whose lives you have either entered into or already begun amongst. The kind of evidence that arises out of its detailed description allows your study to bring into view certain types of phenomena that are too complex for methodologies that seek and detect general features. While I have emphasized how participant observation is concerned with the details, it returns those details to bodies of studies and theoretical approaches, (e.g. landscape, gender, children's geographies, more-than-representational theory, performativity, critical realism etc.). Like other methods in human geography, it is through articulating with and altering the ongoing work of these approaches that participant observation realizes it value.

\section{Summary}

- Participant observation is a method that gathers local and contextualized knowledge of groups, events or practices.

- Insider knowledge can be gathered about places we are already familiar with (and thus are insiders already) or places that are at a distance from our current situation which require us to become competent in new practices, ways of understanding the world and local moral orders

- Note-taking and noticing is essential from the beginning to the end of any participant observation and can be aided by bracketing phenomena, turning things into instructions and breaching experiments

- Participant observation supports the translation of know-how and exploring cultural difference and similitude.

Key reading 


\section{The following books and articles provide good examples of participant observation in}

practice:

- Crang P (1994) is an example of participant observation used to examine how a waiter's work gets done and that by looking closely at this work we can learn about surveillance and display in workplaces

- Harper et al. (2000) is based on two of the authors spending time working alongside the employees of new telephone-banking facilities and traditional banks and building societies.

- Parr (1998) successfully accesses and helps us understand a vulnerable group within the city using covert participant observation.

- Vannini (2012) is an ambitious, extensive and evocatively written participant observation of ferry travel on the West Coast of Canada. There is also a parallel website: http://ferrytales.innovativeethnographies.net

- Venkatesh (2009) is a popular participant observation study of black urban gangs. Venkatesh was originally held hostage by the gang before later being allowed to become a legitimate peripheral participant.

Note: Full details of the above can be found in the references list below.

\section{References}

Cochoy, F. (2008) 'Calculation, qualculation, calqulation: shopping cart arithmetic, equipped cognition and the clustered consumer'. Marketing Theory, 8(1), 15.

Crang, P. (1994) 'It's showtime: on the workplace geographies of display in a restaurant in South East England', Environment and Planning D: Society and Space, 12: 675-704.

Gordon, R. J. (2012) Ordering Networks: Motorways and the Work of Managing Disruption. PhD Thesis University of Durham, Durham.

Hall, T., \& Smith, R. J. (2013) 'Knowing the city: maps, mobility and urban outreach work'. Qualitative Research, 14(3), 294-310.

Harper, R., Randall, D. and Rouncefield, M. (2000) Organisational Change and Retail Finance: An Ethnographic Perspective. London: Routledge. 
Hirschauer, S. (2005) 'On Doing Being a Stranger: The Practical Constitution of Civil Inattention'. Journal for the Theory of Social Behaviour, 35(1), 41-67.

Katz, J. (2001) 'From How to Why: On luminous description and causal inference in ethnography (Part 1)'. Ethnography, 2(4), 443-473.

Katz, J. (2002) 'From how to why: On luminous description and causal inference in ethnography (Part 2)'. Ethnography, 3(1), 64-90.

Latour, B. (2010). The Making of Law - An Ethnography of the Conseil d'Etat. Cambridge: Polity Press.

Laurier, E., Lorimer, H., Brown, B., Jones, O., Juhlin, O., Noble, A., Perry, M., Pica, D., Sormani, P., Strebel, I., Swan, L., Taylor, A., Watts, L., \& Weilnemann, A. (2008). Driving and "Passengering”: Notes on the Ordinary Organization of Car Travel. Mobilities, 3(1), 1-24.

Laurier, E. (2008) 'How breakfast happens in the café', Time and Society, 17: 119-143.

Lave, J., \& Wenger, E. (1991) Situated Learning. Cambridge: Cambridge University Press.

Leidner, R. (1993). Fast Food, Fast Talk: service work and the routinization of everyday life. Berkeley, CA: University of California Press.

Parr, H. (1998) 'Mental health, ethnography and the body'. Area, 30(1): 28-37.

Throsby, K. (2013). "If I go in like a cranky sea lion, I come out like a smiling dolphin”: marathon swimming and the unexpected pleasures of being a body in water. Feminist Review, 103(0), 5-22.

Saldanha, A. (2007). Psychedelic White: Goa Trance and the Viscosity of Race. Minneapolis: University of Minnesota Press.

Vannini, P. (2012) Ferry Tales: Mobility, Place, and Time on Canada's West Coast. London: Routledge.

Venkatesh, S. (2009). Gang Leader for a Day. Harmondsworth: Penguin.

Wright, M. W. (2001). Desire and the Prosthetics of Supervision: A Case of Maquiladora Flexibility. Cultural Anthropology, 16(3), 354-373. 
Wylie, J. (2005) 'A single day's walking: narrating self and landscape on the South West Coast Path. Transactions of the Institute of British Geographers,30 (2): 234-47 